\title{
ARTICLE
}

Received 16 Apr 2012 | Accepted 14 Dec 2012 | Published 22 Jan 2013 DOl: 10.1038/ncomms2387

\section{Creation of helical Dirac fermions by interfacing two gapped systems of ordinary fermions}

Z.F. Wang ${ }^{1, \star}$, Meng-Yu Yao ${ }^{2, \star}$, Wenmei Ming ${ }^{1}$, Lin Miao² ${ }^{2}$ Fengfeng Zhu ${ }^{2}$, Canhua Liư ${ }^{2}$, C.L. Gao ${ }^{2}$, Dong Qian², Jin-Feng $\mathrm{Jia}^{2} \&$ Feng Liu ${ }^{1}$

Topological insulators are a unique class of materials characterized by a Dirac cone state of helical Dirac fermions in the middle of a bulk gap. When the thickness of a three-dimensional topological insulator is reduced, however, the interaction between opposing surface states opens a gap that removes the helical Dirac cone, converting the material back to a normal system of ordinary fermions. Here we demonstrate, using density function theory calculations and experiments, that it is possible to create helical Dirac fermion state by interfacing two gapped films-a single bilayer $\mathrm{Bi}$ grown on a single quintuple layer $\mathrm{Bi}_{2} \mathrm{Se}_{3}$ or $\mathrm{Bi}_{2} \mathrm{Te}_{3}$. These extrinsic helical Dirac fermions emerge in predominantly Bi bilayer states, which are created by a giant Rashba effect with a coupling constant of $\sim 4 \mathrm{eV} \cdot \AA$ due to interfacial charge transfer. Our results suggest that this approach is a promising means to engineer topological insulator states on non-metallic surfaces.

\footnotetext{
${ }^{1}$ Department of Materials Science and Engineering, University of Utah, Salt Lake City, Utah 84112, USA. ${ }^{2}$ Key Laboratory of Artificial Structures and Quantum Control (Ministry of Education), Department of Physics, Shanghai Jiao Tong University, Shanghai 200240, China. * These authors contributed equally to this work. Correspondence and requests for materials should be addressed to D.Q. (email: dqian@sjtu.edu.cn) or to F.L. (email: fliu@eng.utah.edu).
} 
T opological insulators (TIs) have attracted a great deal of recent interest as a new class of materials ${ }^{1-9}$. One unique property of TIs is its electronic structure characterized by the helical Dirac cone states residing in the middle of a bulk insulating gap. Charge carriers in such Dirac states are helical Dirac fermions (HDFs), which, different from ordinary fermions, are massless relativistic particles with their spin locked to their momentum ideally in perpendicular direction. On one hand, the HDFs are expected to give rise to a range of fundamentally new physical phenomena, such as anomalous half-integer quantum Hall effect ${ }^{10,11}$, Majorana Fermions ${ }^{12}$ and fractionally charged quantum particles ${ }^{13}$. On the other hand, the spin transport of HDFs is protected against back scattering by time-reversal symmetry, which affords a promising paradigm of spintronic devices.

So far, the HDFs are considered to be an intrinsic property of TIs, which only exist in TIs with sufficient size. If the dimension of three-dimentional TIs is reduced, the coupling between the surface states coupled with quantum confinement effect will open an energy gap, eliminating the HDF states, that is, the linear Dirac bands of HDFs revert to the parabolic conventional bands of normal fermions upon gap opening ${ }^{14-16}$. For example, when the thickness of $\mathrm{Bi}_{2} \mathrm{Se}_{3}$ (or $\mathrm{Bi}_{2} \mathrm{Te}_{3}$ ), two well-known threedimensional TIs, is reduced to less than six quintuple layers (QLs), a gap opens ${ }^{14-16}$. While the ultrathin Bi (111) films of less than four bilayers (BLs), a two-dimensional TI system, is characterized with a finite gap ${ }^{17-20}$. Therefore, the common expectation is that the HDFs cannot survive in the gapped systems.

Here we report the discovery of extrinsic formation of HDFs by interfacing two gapped thin films: a single BL Bi (111) film grown on a single $\mathrm{QL} \mathrm{Bi}_{2} \mathrm{Se}_{3}$ or $\mathrm{Bi}_{2} \mathrm{Te}_{3}$ substrate. Using density function theory (DFT) band calculations, we predict the existence of helical Dirac cone in such supposedly ordinary fermion systems and the prediction is directly confirmed by experiment. Further theoretical analysis shows that the extrinsically created helical Dirac cone consists of predominantly Bi bilayer states. It is induced by a giant Rashba effect of band splitting with a coupling constant up to $\sim 4 \mathrm{eV} \AA$, resulting from the internal electric field due to interfacial charge transfer. Our findings provide a promising new method to manipulate the electronic and topological states by interface engineering.

\section{Results}

Construction of two gapped systems. Our idea of interfacing two gapped films of single BL Bi (111) film and single $\mathrm{QL} \mathrm{Bi}_{2} \mathrm{Se}_{3}$ or $\mathrm{Bi}_{2} \mathrm{Te}_{3}$ is partly motivated by existing knowledge of growing $\mathrm{Bi}$ film on different substrates. It is known that when Bi grown on metal $^{21,22}$ or semiconductor substrates (for example, $\left.\mathrm{Si}, \mathrm{Ge}\right)^{23-25}$, an extrinsic helical Dirac surface state can be created by the substrate induced spin-splitting of Bi bands, that is, the Rashba effect. However, generally there exist strong interactions between the $B i$ and the substrate leading to strong hybridization of surface and bulk states, and additionally metal substrate is undesirable for device applications. Recent experiments have grown Bi (111) bilayer on $\mathrm{TI} \mathrm{Bi}_{2} \mathrm{Te}_{3}$ (refs 26,27) and $\mathrm{Bi}_{2} \mathrm{Se}_{3}$, which also see extrinsic formation of the helical Dirac states from Bi. However, the problem remains that there exists the intrinsic Dirac state and other bulk states of $\mathrm{Bi}_{2} \mathrm{Te}_{3}$ substrate that can hybridize with the extrinsic surface Dirac state of $\mathrm{Bi} \mathrm{BL}^{27}$. Therefore, one needs to find a suitable gapped substrate for growing Bi that will avoid strong hybridization between the extrinsic Bi Dirac states and substrate states, so as to better isolate surface Dirac transport. In this regard, growing $\mathrm{Bi}$ bilayer on a gapped $1 \mathrm{QL} \mathrm{Bi}_{2} \mathrm{Te}_{3}$ (or $\mathrm{Bi}_{2} \mathrm{Se}_{3}$ ) substrate versus on a semimetal thick (>6 QL) $\mathrm{Bi}_{2} \mathrm{Te}_{3}$ (or $\mathrm{Bi}_{2} \mathrm{Se}_{3}$ ) substrate makes a significant difference. Further, experimentally growing high quality $1 \mathrm{QL} \mathrm{Bi}_{2} \mathrm{Te}_{3}\left(\right.$ or $\left.\mathrm{Bi}_{2} \mathrm{Se}_{3}\right)$ film turns out to be much harder than thick films, as we discuss below.

DFT band structures. A single BL Bi (111) film is predicted to be a gapped two-dimensional $\mathrm{TI}^{17-20}$. Our calculations show that at the equilibrium substrate lattice constant of $\mathrm{Bi}_{2} \mathrm{Se}_{3}$ and $\mathrm{Bi}_{2} \mathrm{Te}_{3}$, the strained Bi (111) film has an energy gap of $\sim 0.17 \mathrm{eV}$ (Fig. 1a) and $\sim 0.44 \mathrm{eV}$ (Supplementary Fig. S1a), respectively. On the other hand, $\mathrm{Bi}_{2} \mathrm{Se}_{3}$ and $\mathrm{Bi}_{2} \mathrm{Te}_{3}$ are well-known three-dimentional TIs with a single helical Dirac cone below Fermi level. However, when their thickness is reduced to $<6 \mathrm{QL}$, a finite energy gap opens removing the Dirac cone. As shown in Fig. $1 \mathrm{~b}$ and Supplementary Fig. S1b, we can clearly see an energy gap in $1 \mathrm{QL}$ $\mathrm{Bi}_{2} \mathrm{Se}_{3}(\sim 0.5 \mathrm{eV})$ and $\mathrm{Bi}_{2} \mathrm{Te}_{3}(\sim 0.3 \mathrm{eV})$, which are consistent with previous works ${ }^{15,16}$. After $1 \mathrm{BL} \mathrm{Bi}(111)$ is deposited onto $1 \mathrm{QL} \mathrm{Bi}_{2} \mathrm{Se}_{3}\left(\right.$ or $\mathrm{Bi}_{2} \mathrm{Te}_{3}$ ), the band structures are found to change dramatically. Most surprisingly, the gap vanishes as a linear dispersive band emerges around the $\Gamma$ point to form the Dirac cone, marked with 'D' in Fig. 1c,d and Supplementary Fig. S1c,d. For the $\mathrm{Bi} / \mathrm{Bi}_{2} \mathrm{Se}_{3}$ (Fig. 1c,d), the Dirac point locates at $\sim 0.1 \mathrm{eV}$ below the Femi level with a Fermi velocity $\left(V_{\mathrm{F}}\right)$ $\sim 5.3 \times 10^{5} \mathrm{~m} \mathrm{~s}^{-1}$; for the $\mathrm{Bi} / \mathrm{Bi}_{2} \mathrm{Te}_{3}$ (Supplementary Fig. $\mathrm{S} 1 \mathrm{c}, \mathrm{d})$, the Dirac point locates at $\sim 0.2 \mathrm{eV}$ below the Femi level with a $V_{\mathrm{F}} \sim 4.5 \times 10^{5} \mathrm{~m} \mathrm{~s}^{-1}$. We note that the extrinsic Dirac point formed in our system is not perfect as the second derivative of $E(k)$ doesn't exactly vanish. However, the $E(k)$ is almost linear with spin-momentum locking property, having a very large Fermi velocity comparable to that in graphene or other TIs. Therefore, from charge and spin transport point of view, our system works equivalently well for spintronics device applications.

Angle-resolved photoemission spectroscopy band structures. Our DFT predictions are directly confirmed by experiments, as
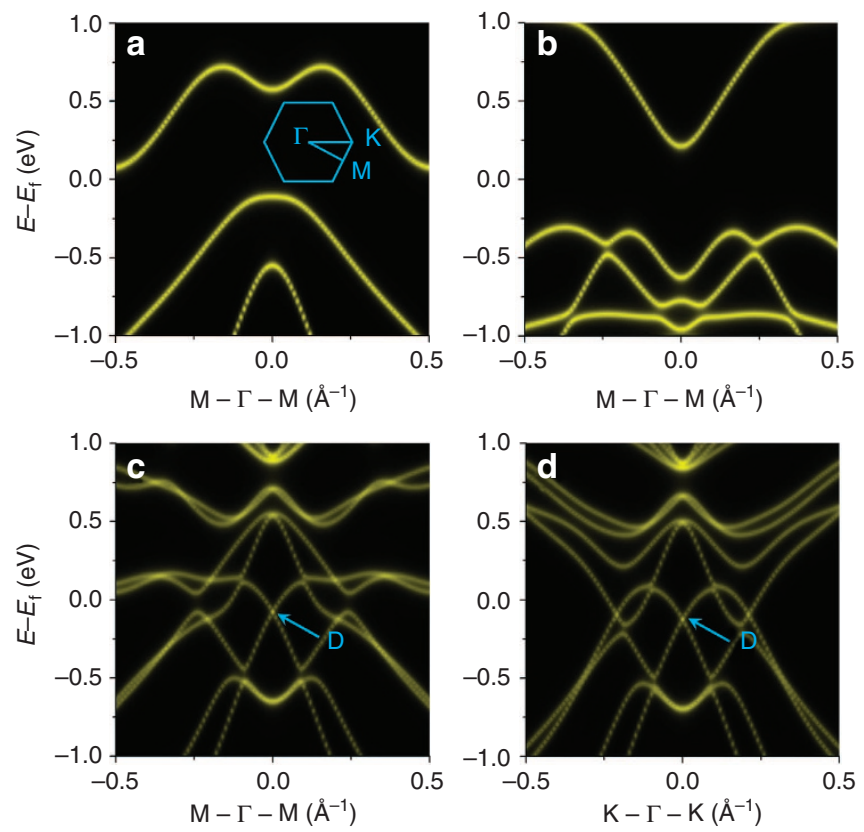

Figure 1 | Theoretical bands along the high-symmetry directions. (a) Bare $\mathrm{Bi}$ (111) $\mathrm{BL}$ along $\mathrm{M}-\Gamma-\mathrm{M}$ direction at the bulk $\mathrm{Bi}_{2} \mathrm{Se}_{3}$ lattice constant. The inset is the first Brillouin zone with high symmetric points of $\Gamma, \mathrm{M}$ and $\mathrm{K}$. (b) Bare $1 \mathrm{QL} \mathrm{Bi} \mathrm{Se}_{3}$ along $\mathrm{M}-\Gamma-\mathrm{M}$ direction. (c,d) $1 \mathrm{BL} \mathrm{Bi}(111)$ on $1 \mathrm{QL} \mathrm{Bi} \mathrm{Se}_{3}$ along $\mathrm{M}-\Gamma-\mathrm{M}$ and $\mathrm{K}-\Gamma-\mathrm{K}$ directions, respectively. The newly created Dirac cone is marked with ' $D$ '. The relative intensity of bands at different $k$-points scales with the number of local density of states. 

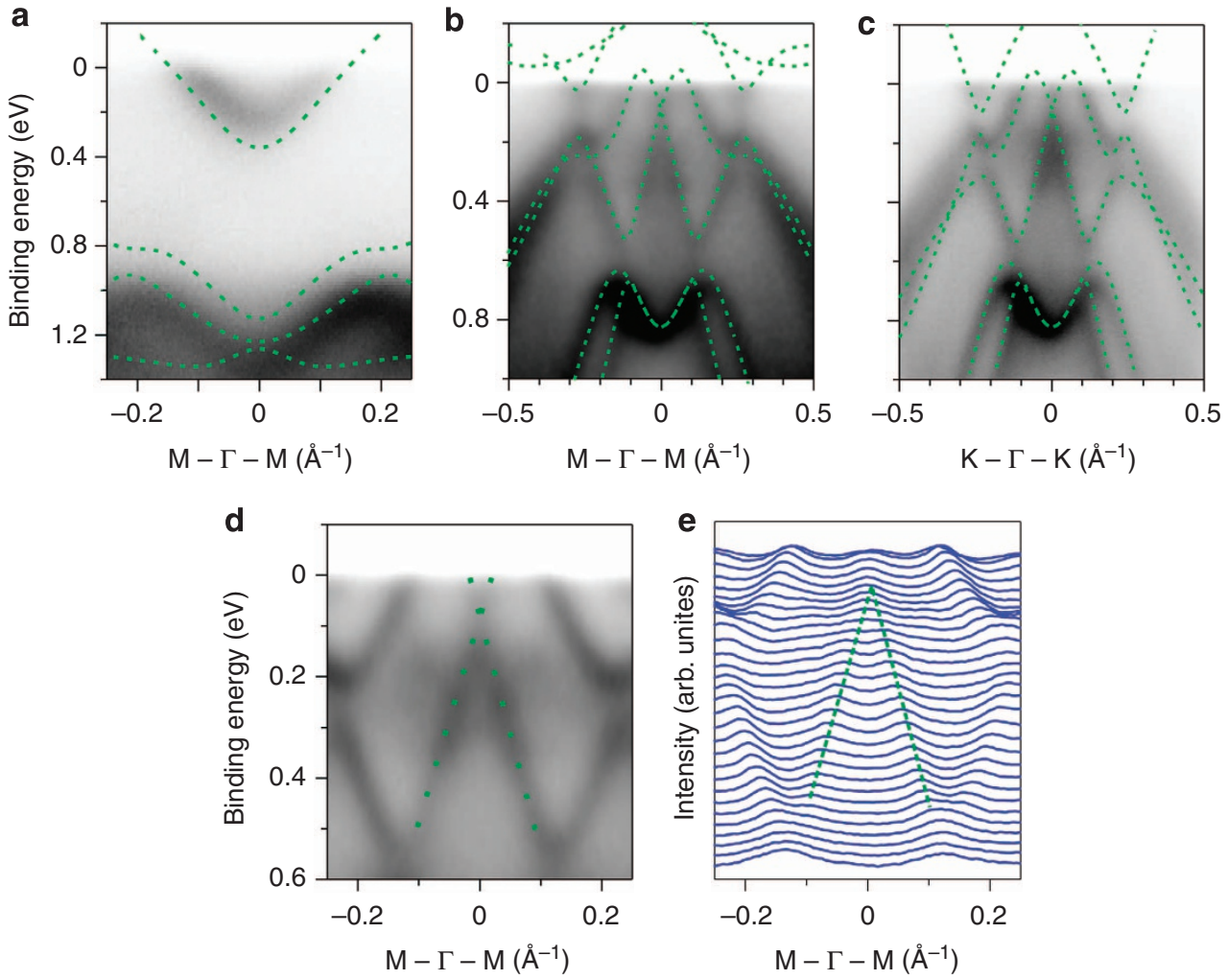

Figure 2 | Experimental bands superimposed with theoretical bands. (a) Bare $1 \mathrm{QL} \mathrm{Bi} \mathrm{Se}_{3}$. (b) and (c) $1 \mathrm{BL} B \mathrm{Bi}(111)$ on $1 \mathrm{QL} \mathrm{Bi} \mathrm{Se}_{3}$ along $M-\Gamma-\mathrm{M}$ and $\mathrm{K}-\Gamma-\mathrm{K}$ directions, respectively. Green dashed lines are the theoretical bands. (d,e) High resolution ARPES spectra near the Dirac cone and corresponding MDC curves. Green dotted line marks the linearly dispersive bands that crossing at Dirac point at $\sim 70$ meV blew Fermi level. Note that the calculated bands are slightly different from Fig. 1 because different lattice parameter used.

shown in Fig. 2. A single BL Bi (111) is grown on the (111)oriented $1 \mathrm{QL} \mathrm{Bi}_{2} \mathrm{Se}_{3}$ substrates, which were grown first on graphene prepared on $\mathrm{SiC}$ wafer ${ }^{28}$. We found that growth of high quality ultrathin film of $1 \mathrm{QL} \mathrm{Bi}_{2} \mathrm{Se}_{3}$ or $\mathrm{Bi}_{2} \mathrm{Te}_{3}$ turned out to be much harder than growth of thick film of multi-QLs. So far, we only attained high quality of $1 \mathrm{QL} \mathrm{Bi}_{2} \mathrm{Se}_{3}$ film to our satisfaction, but not for $1 \mathrm{QL} \mathrm{Bi}_{2} \mathrm{Te}_{3}$. The thickness and quality of the films are monitored by in situ reflection high-energy electron diffraction and scanning tunnelling microscopy (STM). The electronic bands are measured directly by angle-resolved photoemission spectroscopy (ARPES). In Fig. 2a, an energy gap is observed for the bare $1 \mathrm{QL} \mathrm{Bi}_{2} \mathrm{Se}_{3}$. Bi can grow epitaxial with a unit of $\mathrm{BL}$ along (111) orientation on $\mathrm{Bi}_{2} \mathrm{Se}_{3}$. After deposition of a single BL Bi (111), the spectra are dramatically changed and a Dirac cone clearly appears around the $\Gamma$ point, as shown in Fig. $2 \mathrm{~b}, \mathrm{c}$. The DFT bands calculated at the experimental graphene lattice constant (green dashed lines) are overlaid with the ARPES spectra along high symmetric directions and the agreement between the theory and experiment is very good. In order to match experimental data, the Fermi level of DFT bands is slightly shifted. The Dirac cone is isotropic. Fig. 2d,e presents the high resolution ARPES spectra near the Dirac cone and corresponding momentum distribution curves. The linearly dispersive bands of the Dirac cone are clearly resolved. The Dirac point is about $0.07 \mathrm{eV}$ below Fermi level estimated from the crossing point of the linearly dispersive bands (green dotted line). The $V_{\mathrm{F}}$ of the Dirac cone is $\sim 6 \times 10^{5} \mathrm{~ms}^{-1}$, which is the same as that of bare $\mathrm{Bi}_{2} \mathrm{Se}_{3}$.

Extrinsic helical Dirac states. We have also confirmed the helical properties of the newly formed extrinsic Dirac cones in the interfaced systems of a single $\mathrm{BL} \mathrm{Bi}(111)$ and $1 \mathrm{QL} \mathrm{Bi}_{2} \mathrm{Se}_{3}$ (or $\mathrm{Bi}_{2} \mathrm{Te}_{3}$ ), which are individually gapped materials. HDFs are characterized by the spin-momentum locking relations ${ }^{29}$ : (i) the spin direction is perpendicular to the momentum direction; (ii) the spins have the opposite directions at the inverse momenta. We have calculated three spin components of the Bloch state in the Brillouin zone, as shown in Fig. 3. Figure 3a shows the zoomin band structure around the Dirac cone for the $\mathrm{Bi} / \mathrm{Bi}_{2} \mathrm{Se}_{3}$ system, indicating two equal-energy cuts (circles) above and below the $\Gamma$ point with $k=0.04 \AA^{-1}$ (radius of circle). The in-plane spin projections along the upper and lower circles are plotted in Fig. $3 c, d$, respectively. The spins point clockwise around the upper circle, but counterclockwise around the lower circle, and they are in opposite directions at the inverse momenta. In addition, the spins are not completely in-plane, and their out-ofplane projections are plotted in Fig. 3e, which oscillate periodically around circle with a period of $120^{\circ}$ (reflecting the threefold rotational symmetry) and have the opposite phase in the upper and lower circles. Thus, as the electrons trace the circle in momentum space, their spins lie almost in the plane of the circle, but wobble above and below the plane periodically. The finite out-of-plane spin-component is due to the warping effect of the Dirac cone (see Fig. 3a), which has not a perfect linear dispersion. The six nodal points in the out-of-plane wobbling motion (Fig. 3e) correspond to high-symmetry $\Gamma-\mathrm{M}$ directions. We also calculated the angle $(\alpha)$ between the electron spin and momentum direction, as shown in Fig. 3f. $\alpha$ is very small, $<0.3^{\circ}$ and $0.1^{\circ}$ in the upper and lower circle, respectively, indicating that spins are almost perpendicular to their momenta. All these features demonstrate the spin-momentum locking property of the helical Dirac states. The same results and conclusions are drawn for the $\mathrm{Bi} / \mathrm{Bi}_{2} \mathrm{Te}_{3}$ system, as shown in Supplementary Fig. S2. 

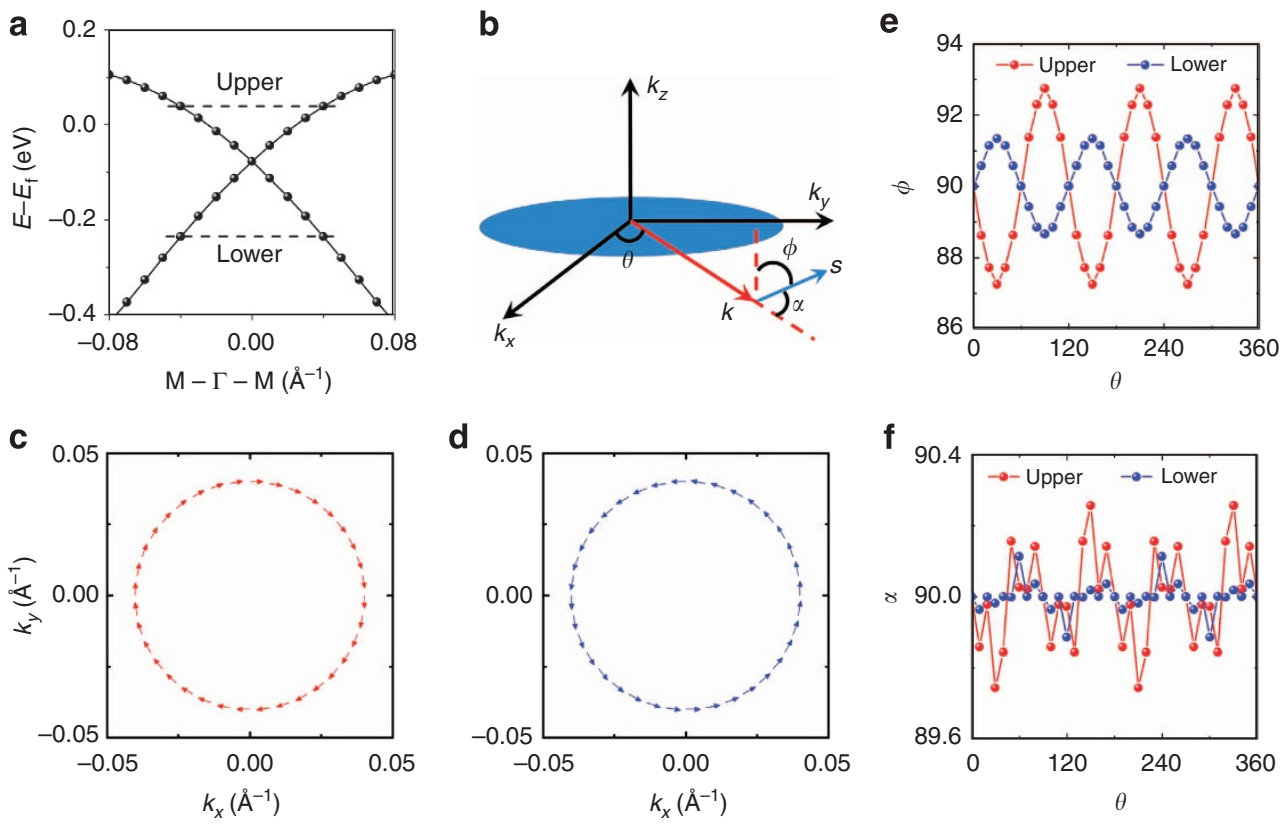

Figure 3 | Helical states near the Dirac cone. (a) Two equal-energy cuts as marked above and below Dirac point for calculating the helical Dirac states in $\mathrm{Bi} / \mathrm{Bi}_{2} \mathrm{Se}_{3}$. (b) Definitions of the $\theta$ (angle between $k$ and $k_{x}$ axis), $\phi$ (angle between $S$ and $k_{z}$ axis) and $\alpha$ (angle between $k$ and $S$ ), which are used to characterize the spin direction $(S)$ with respect to the momentum direction $(k)$. (c,d) In-plane spin-momentum relation in the upper and lower cut in (a), respectively. (e) Out-of-plane spin-momentum relation in the upper and lower cuts in (a). (f) Angle between spin and momentum in the upper and lower cuts in (a).

\section{Discussion}

Next, we explain where the Dirac cone states come from, the $1 \mathrm{BL}$ $\mathrm{Bi}$ or $1 \mathrm{QL} \mathrm{Bi}_{2} \mathrm{Se}_{3}$ (or $\mathrm{Bi}_{2} \mathrm{Te}_{3}$ )? Previous studies ${ }^{18}$ showed that the Bi (111) film represents a special class of system having an intermediate inter-bilayer coupling strength, which has a significant influence on its topological property. At equilibrium, the interfacial distance $(d)$ between $\mathrm{Bi} \mathrm{BL}$ and $\mathrm{Bi}_{2} \mathrm{Se}_{3} \mathrm{QL}$ is $\sim 3 \AA$ and the interface energy is $\sim 0.16 \mathrm{eV}$ per unit cell, which are noticeably larger than the typical values of van der Waals bond but smaller than those of typical chemical bond. To gain some insight about the influence of the interfacial coupling on the helical spin states, we have performed a set of model DFT calculations by artificially increasing the interfacial distance between the $\mathrm{Bi} \mathrm{BL}$ and $\mathrm{Bi}_{2} \mathrm{Se}_{3} \mathrm{QL}$ to gradually tune the interface coupling strength ${ }^{18}$, as shown in Fig. 4. At a large interfacial distance (for example, $d \sim 6 \AA$ in Fig. 4a), without the interfacial coupling, the band structure would be a simple superposition of the bare Bi BL and $\mathrm{Bi}_{2} \mathrm{Se}_{3}$ QL bands. However, we observe a slight band splitting in the double-degenerated $\mathrm{Bi}$ and $\mathrm{Bi}_{2} \mathrm{Se}_{3}$ band. With the decreasing interfacial distance, the increased interfacial coupling continues to further split and reshape the bands, leading eventually to the formation of helical Dirac cone. By analysing the spectral components of Dirac cone states during this process, we found that the Dirac cone is predominantly coming from the Bi BL, with $\sim 95 \%$ spectral weight of Bi BL states at the equilibrium distance, as shown in Fig. 4c. Similar results are found for the interface between $\mathrm{Bi} \mathrm{BL}$ and $\mathrm{Bi}_{2} \mathrm{Te}_{3} \mathrm{QL}$, with $\sim 90 \% \mathrm{Bi} \mathrm{BL}$ states in the helical Dirac cone at the equilibrium distance, as shown in Supplementary Fig. S3a-c.

Lastly, we will address how the Dirac cone states are formed. From the above calculations and analyses, we learnt that the extrinsic HDFs originate from the $\mathrm{Bi} \mathrm{BL}$, while the $\mathrm{QL}$ of $\mathrm{Bi}_{2} \mathrm{Se}_{3}$ (or $\mathrm{Bi}_{2} \mathrm{Te}_{3}$ ) substrate provides possibly a triggering mechanism that induces the Dirac cone states in Bi BL. We also know that Bi (111) BL has a strong spin-orbit coupling, and by itself its bands are double-degenerate conserving the inversion symmetry.
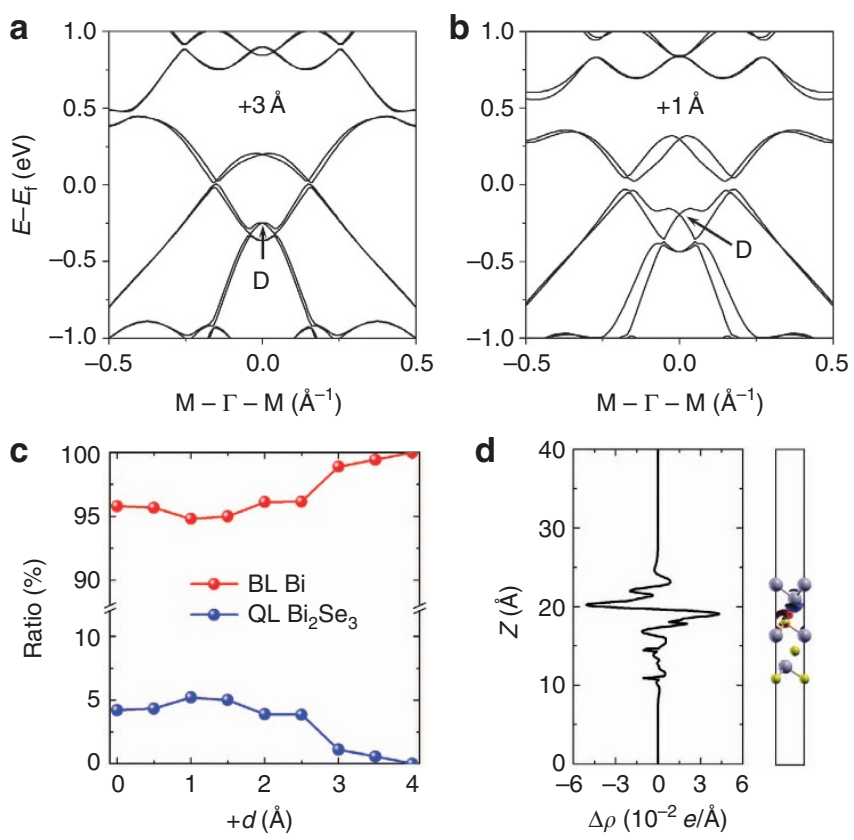

Figure 4 | Model DFT bands as a function of interfacial distance.

$(\mathbf{a}, \mathbf{b})$ Bands at the artificially increased interfacial distance by 3 and $1 \AA$, respectively, showing the creation process of helical Dirac cone. (c) Spectral weight at the Dirac point resolved between the $\mathrm{Bi} B \mathrm{BL}$ and $\mathrm{Bi}_{2} \mathrm{Se}_{3} \mathrm{QL}$ as a function of interfacial distance. (d) Interfacial charge transfer between $\mathrm{Bi} \mathrm{BL}$ and $\mathrm{Bi}_{2} \mathrm{Se}_{3} \mathrm{QL}$ at the equilibrium distance, showing the internal electrical field.

Deposition of $\mathrm{Bi} \mathrm{BL}$ on $\mathrm{Bi}_{2} \mathrm{Se}_{3}$ (or $\mathrm{Bi}_{2} \mathrm{Te}_{3}$ ) will break such symmetry, and hence lift the band degeneracy. However, this effect of symmetry breaking is limited, likely insufficient to induce 
a band splitting and reshaping as dramatic as we have observed. To further understand the interaction between the Bi BL and $\mathrm{Bi}_{2} \mathrm{Se}_{3}$ (or $\mathrm{Bi}_{2} \mathrm{Te}_{3}$ ), we calculated the differential charge density at the interface, defined as $\rho_{\text {diff }}=\rho_{\mathrm{BL}+\mathrm{QL}}-\rho_{\mathrm{BL}}-\rho_{\mathrm{QL}}$, as shown in Fig. $4 \mathrm{~d}$ (see also Supplementary Fig. $\mathrm{S} 3 \mathrm{~d}$ for $\mathrm{Bi} / \mathrm{Bi}_{2} \mathrm{Te}_{3}$ ). Clearly, a substantial charge transfer at the interface has occurred. The $\mathrm{Bi}$ $\mathrm{BL}$ acts as a donor and the $\mathrm{Bi}_{2} \mathrm{Se}_{3}$ (or $\mathrm{Bi}_{2} \mathrm{Te}_{3}$ ) as an acceptor, with the electrons transferring from the former to the latter. This charge transfer generates a large internal electric field at the interface region, with a field direction pointing from the Bi BL to the $\mathrm{Bi}_{2} \mathrm{Se}_{3}$ (or $\mathrm{Bi}_{2} \mathrm{Te}_{3}$ ), and field intensity estimated as high as $\sim 1 \mathrm{~V} / \AA$. Thus, we propose this phenomenon is very similar to the external electric field (or band bending) effect that induces Rashba band splitting in the surface state of $\mathrm{TIs}^{30-32}$. We estimated the Rashba coupling constant (using the twodimentional free electron gas model) from the calculated band splitting at the equilibrium structures, which is $\sim 4 \mathrm{eV} \cdot \AA$. Therefore, the HDFs are extrinsically created by a giant Rashba effect due to the interfacial charge transfer.

To further support the charge transfer induced Rashba effect is the dominated factor for realizing the extrinsic helical Dirac states, we also studied the band structure for Bi BL on $1 \mathrm{QL} \mathrm{In}_{2} \mathrm{Se}_{3}$ and 5-layer $\mathrm{Cu}$ (111) substrate, as shown in Supplementary Figs S4 and S5, respectively. A clearly charge transfer between the Bi BL and these non-TI substrates (Supplementary Figs. S4i and S5i) can be observed in both cases (one semiconductor and one metal), and the similar Dirac cone states are also generated. However, the $\mathrm{Bi} \mathrm{BL}$ become metallic at the $\mathrm{In}_{2} \mathrm{Se}_{3}$ lattice constant, and the $\mathrm{Cu}$ (111) substrate is also metallic. Thus, comparing to $\mathrm{Bi} / \mathrm{Bi}_{2} \mathrm{Se}_{3}$ and $\mathrm{Bi} / \mathrm{Bi}_{2} \mathrm{Te}_{3}$ system, these Dirac cone states are not within the gapped region of $\mathrm{Bi} / \mathrm{In}_{2} \mathrm{Se}_{3}$ and $\mathrm{Bi} / \mathrm{Cu}$. The previous studies for Bi grown on $\mathrm{Si}$ (111) only showed the monomer or trimer Bi configuration on the substrate, which cannot maintain the intrinsic structure of the Bi BL.

\section{Methods \\ Calculation method. DFT calculations for a single BL Bi (111) on a single QL $\mathrm{Bi}_{2} \mathrm{Se}_{3}$ and $\mathrm{Bi}_{2} \mathrm{Te}_{3}$ are carried out in the framework of the Perdew-Burke- Ernzerhof-type generalized gradient approximation using VASP package ${ }^{33}$. The experimental bulk lattice constants of the substrate are used $\left(a=4.138 \AA\right.$ for $\mathrm{Bi}_{2} \mathrm{Se}_{3}$ and $a=4.386 \AA$ for $\mathrm{Bi}_{2} \mathrm{Te}_{3}$ ), and the $\mathrm{Bi} \mathrm{BL}$ is strained to match the substrate lattice constant. All calculations are performed with a plane-wave cutoff of $400 \mathrm{eV}$ on an $11 \times 11 \times 1$ Monkhorst-Pack $k$-point mesh, and the vacuum layer is over $20 \AA$ thick to ensure decoupling between neighbouring slabs. During structural relaxation, all the atoms are allowed to relax until the forces are smaller than $0.01 \mathrm{eV} / \AA$. To reproduce the ARPES band for $\mathrm{Bi} / \mathrm{Bi}_{2} \mathrm{Se}_{3}$ in Fig. 2, we use a larger lattice constant $a=4.37 \AA$, which can match the graphene/SiC wafer in the experiment.}

Experiment method. Flat $\mathrm{Bi}_{2} \mathrm{Se}_{3}$ single QL films is grown by molecular beam epitaxy method on graphene/SiC wafer and on highly ordered pyrolytic graphite substrates. Bi films are deposited on $\mathrm{Bi}_{2} \mathrm{Se}_{3}$ at $200 \mathrm{~K}$ and then annealed at room temperature. The thickness of $\mathrm{Bi}_{2} \mathrm{Se}_{3}$ and $\mathrm{Bi}$ films is monitored by reflection highenergy electron diffraction oscillation and scanning tunnelling microscopy. The sample temperature is kept at $100 \mathrm{~K}$ during measurement. ARPES measurements are performed with in-lab He discharge lamp (He-I 21.2 eV). Energy resolution is better than $25 \mathrm{meV}$ and angular resolution is better than $0.02 \AA^{-1}$.

\section{References}

1. Kane, C. L. \& Mele, E. J. Quantum spin hall effect in graphene. Phys. Rev. Lett. 95, 226801 (2005)

2. Bernevig, B. A. \& Zhang, S.-C. Quantum spin hall effect. Phys. Rev. Lett. 96, 106802 (2006)

3. Bernevig, B. A. et al. Quantum spin hall effect and topological phase transition in HgTe Quantum Wells. Science 314, 1757-1761 (2006).

4. Fu, L. \& Kane, C. L. Topological insulators with inversion symmetry. Phys. Rev. B 76, 045302 (2007).

5. Hsieh, D. et al. A tunable topological insulator in the spin helical dirac transport regime. Nature 460, 1101-1105 (2009).

6. Xia, Y. et al. Observation of a large-gap topological-insulator class with a single dirac cone on the surface. Nat. Phys. 5, 398-402 (2009).
7. Zhang, H. J. et al. Topological insulators in $\mathrm{Bi}_{2} \mathrm{Se}_{3}, \mathrm{Bi}_{2} \mathrm{Te}_{3}$ and $\mathrm{Sb}_{2} \mathrm{Te}_{3}$ with a single Dirac cone on the surface. Nat. Phys. 5, 438-442 (2009).

8. Chen, Y. L. et al. Experimental realization of a three-dimensional topological insulator $\mathrm{Bi}_{2} \mathrm{Te}_{3}$. Science 325, 178-181 (2009).

9. Hasan, M. Z. \& Kane, C. L. Topological insulators. Rev. Mod. Phys. 82, 3045-3067 (2010).

10. Fu, L. et al. Topological insulators in three dimensions. Phys. Rev. Lett. 98, 106803 (2007).

11. Qi, X.-L. et al. Topological field theory of time-reversal invariant insulators. Phys. Rev. B 78, 195424 (2008).

12. Akhmerov, A. R. et al. Electrically detected interferometry of majorana fermions in a topological insulator. Phys. Rev. Lett. 102, 216404 (2009).

13. Seradjeh, B. et al. Exciton condensation and charge fractionalization in a topological insulator film. Phys. Rev. Lett. 103, 066402 (2009).

14. Zhang, Y. et al. Crossover of the three-dimensional topological insulator $\mathrm{Bi}_{2} \mathrm{Se}_{3}$ to the two-dimensional limit. Nat. Phys. 6, 584-588 (2010).

15. Park, K. et al. Robustness of topologically protected surface states in layering of $\mathrm{Bi}_{2} \mathrm{Te}_{3}$ thin films. Phys. Rev. Lett. 105, 186801 (2010).

16. Yazyev, O. V. et al. Spin polarization and transport of surface states in the topological insulators $\mathrm{Bi}_{2} \mathrm{Se}_{3}$ and $\mathrm{Bi}_{2} \mathrm{Te}_{3}$ from first principles. Phys. Rev. Lett. 105, 266806 (2010).

17. Murakami, S. Quantum spin hall effect and enhanced magnetic response by spin-orbit coupling. Phys. Rev. Lett. 97, 236805 (2006).

18. Liu, Z. et al. Stable nontrivial $Z_{2}$ topology in ultrathin Bi (111) films: a firstprinciples study. Phys. Rev. Lett. 107, 136805 (2011).

19. Koroteev, Y. M. et al. First-principles investigation of structural and electronic properties of ultrathin Bi films. Phys. Rev. B 77, 045428 (2008).

20. Wada, M. et al. Localized edge states in two-dimensional topological insulators: ultrathin Bi films. Phys. Rev. B 83, 121310(R) (2011).

21. Ast, C. R. et al. Giant spin splitting through surface alloying. Phys. Rev. Lett. 98, 186807 (2007).

22. Mathias, S. et al. Quantum-well-induced giant spin-orbit splitting. Phys. Rev. Lett. 104, 066802 (2010).

23. Gierz, I. et al. Silicon surface with giant spin splitting. Phys. Rev. Lett. 103, 046803 (2009)

24. Ohtsubo, Y. et al. Spin-polarized semiconductor surface states localized in subsurface layers. Phys. Rev. B. 82, 201307(R) (2010).

25. Hatta, S. \& Aruga, T. Large rashba spin splitting of surface resonance bands on semiconductor surface. Phys. Rev. B. 80, 113309 (2009).

26. Hirahara, T. et al. Interfacing $2 \mathrm{D}$ and $3 \mathrm{D}$ topological insulators: $\mathrm{Bi}$ (111) bilayer on $\mathrm{Bi}_{2} \mathrm{Te}_{3}$. Phys. Rev. Lett. 107, 166801 (2011).

27. Yang, F. et al. Spatial and energy distribution of topological edge states in single Bi (111) bilayer. Phys. Rev. Lett. 109, 016801 (2012).

28. Song, C.-L. et al. Topological insulator $\mathrm{Bi}_{2} \mathrm{Se}_{3}$ thin films grown on doublelayer graphene by molecular beam epitaxy. Appl. Phys. Lett. 97, 143118 (2010).

29. Zhao, Y. et al. Helical states of topological insulator $\mathrm{Bi}_{2} \mathrm{Se}_{3}$. Nano Lett. 11, 2088-2091 (2011).

30. King, P. D. C. et al. Large tunable rashba spin splitting of a two-dimensional electron gas in $\mathrm{Bi}_{2} \mathrm{Se}_{3}$. Phys. Rev. Lett. 107, 096802 (2011).

31. Zhu, Z.-H. et al. Rashba spin-splitting control at the surface of the topological insulator $\mathrm{Bi}_{2} \mathrm{Se}_{3}$. Phys. Rev. Lett. 107, 186405 (2011).

32. Benia, H. M. et al. Reactive chemical doping of the $\mathrm{Bi}_{2} \mathrm{Se}_{3}$ topological insulator. Phys. Rev. Lett. 107, 177602 (2011).

33. Kresse, G. \& Hafner, J. Ab initio molecular dynamics for liquid metals. Phys. Rev. B 47, 558-561 (1993).

\section{Acknowledgements}

The theoretical work was supported by US DOE-BES (Grant No. DE-FG02-04ER46027) and we thank the CHPC at University of Utah and NERSC for providing the computing resources. The experimental work was supported by National Basic Research Programme of China (Grant No. 2012CB927401, 2011CB921902, 2013CB921902, 2011CB922200), NSFC (Grant No. 91021002, 11174199, 11134008, 11274228), Shanghai Committee of Science and Technology, China (No. 11JC1405000, 11PJ1405200, 12JC1405300), Shanghai Municipal Education Commission (11ZZ17) and SRF for ROCS, SEM. Z.F.W acknowledges additional support from ARL (Cooperative Agreement No. W911NF-12-2 0023). W.M.M. acknowledges additional support from NSF-MRSCE (Grant No. DMR1121252). D.Q. acknowledges additional support from 'ShuGuang' project supported by Shanghai Municipal Education Commission and Shanghai Education Development Foundation and from Programme for Professor of Special Appointment (Eastern Scholar) at Shanghai Institutions of Higher Learning.

\section{Author contributions}

Z.F.W. carried out the theoretical calculations with assistance from W.M.M. and F.L.; M.Y.Y. did the experiments with assistance from L.M., F.F.Z., C.H.L., C.L.G., D.Q. and J.F.J., F.L, D.Q., and J.F.J. were responsible for the overall direction. Z.F.W., F.L. and D.Q. wrote the paper. 


\section{Additional information}

Supplementary Information accompanies this paper at http://www.nature.com/ naturecommunications

Competing financial interests: The authors declare no competing financial interests.
Reprints and permission information is available online at http://npg.nature.com/ reprintsandpermissions/

How to cite this article: Wang, Z.F. et al. Creation of helical Dirac fermions by interfacing two gapped systems of ordinary fermions. Nat. Commun. 4:1384 doi: 10.1038/ncomms2387 (2013). 